Revue internationale d'éducation de Sèvres

\title{
Le système éducatif belge
}

\section{Cécile de Bouttemont}

\section{(2) OpenEdition}

Journals

Édition électronique

URL : https://journals.openedition.org/ries/1466

DOI : $10.4000 /$ ries. 1466

ISSN : 2261-4265

\section{Éditeur}

France Education international

\section{Édition imprimée}

Date de publication : 1 décembre 2004

Pagination : 101-108

ISBN : 978-2-85420-564-0

ISSN : $1254-4590$

\section{Référence électronique}

Cécile de Bouttemont, "Le système éducatif belge », Revue internationale d'éducation de Sèvres [En ligne], 37 | décembre 2004, mis en ligne le 18 novembre 2011, consulté le 05 juillet 2021. URL : http:// journals.openedition.org/ries/1466; DOI : https://doi.org/10.4000/ries.1466

Ce document a été généré automatiquement le 5 juillet 2021

(c) Tous droits réservés 


\title{
Le système éducatif belge
}

\author{
Cécile de Bouttemont
}

1 La Belgique est un État fédéral qui comporte deux sortes d'entités : les communautés, dont l'élément constitutif est la culture et la langue, et les régions dont l'élément déterminant est le territoire. Les communautés sont au nombre de trois, la Communauté française, la Communauté flamande et la Communauté germanophone ${ }^{1}$. Elles se répartissent sur le territoire divisé administrativement en trois régions : la Région wallonne, la Région flamande et la Région de Bruxelles capitale. Cette dernière possède un statut particulier car elle est située en Communauté flamande mais abrite plus de $80 \%$ de francophones. Ainsi le fédéralisme belge repose sur deux piliers, les communautés, et les régions, qui ne peuvent se superposer l'un à l'autre.

2 Cette organisation politique et territoriale complexe est le résultat de l'évolution historique de la nation belge et de conflits d'intérêts générés par des groupes représentant des valeurs socioculturelles différentes. Ces deux phénomènes ont amené les responsables politiques, dans une période récente, à fédérer ces entités pour mieux tenir compte de leurs spécificités. Ainsi la Belgique a connu depuis 1970 quatre réformes ${ }^{2}$ constitutionnelles qui en ont fait l'État fédéral d'aujourd'hui.

3 L'éducation en Belgique a suivi le même processus que l'organisation politique et trouve son fondement actuel dans la réforme constitutionnelle de 1988, appelée Communautarisation. La Constitution a été révisée pour octroyer aux trois Communautés ${ }^{3}$ la quasi-totalité des compétences en matière d'enseignement.

$4 \mathrm{Au}$ regard des différentes études internationales comparatives, le système éducatif belge se caractérise par de fortes disparités de niveau entre les élèves mais aussi entre les établissements scolaires ${ }^{4}$. Dès lors, la recherche d'une plus grande équité entre les élèves apparait comme l'un des défis majeurs auquel est confronté le système éducatif belge. Parallèlement, dans un contexte socio-économique plus difficile, avec la construction européenne, les acteurs politiques et éducatifs doivent faire face à de nouveaux enjeux avec une marge de manœuvre parfois plus étroite. 


\section{Organisation de l'enseignement depuis 1988}

5 Depuis la Communautarisation, l'État fédéral ne conserve que trois prérogatives en matière d'enseignement : la fixation du début et de la fin de la scolarité obligatoire, les conditions minimales pour la délivrance des diplômes et le régime de retraite ${ }^{5}$.

Répartition par réseau des populations scolaires (fondamental et secondaire) en 1996/1997

\begin{tabular}{|l|c|c|c|}
\hline & Communauté & Officiel subventionné & Libre subventionné \\
\hline Com. française & $16 \%$ & $35 \%$ & $49 \%$ \\
\hline Com. flamande & $15 \%$ & $16 \%$ & $69 \%$ \\
\hline Com. germanophone & Non communiqué & Non communiqué & Non communiqué \\
\hline
\end{tabular}

Source : Eurydice.

6 Les compétences en matière éducative sont identiques d'une communauté à l'autre, même si des différences au niveau institutionnel ${ }^{6}$ peuvent modifier cet équilibre. Les responsabilités de l'enseignement, quant à elles, incombent aux ministères de l'Éducation des trois communautés. En pratique, il existe trois ministères en Communauté française (un pour l'enseignement supérieur et la recherche scientifique, un pour l'enseignement fondamental et un pour l'enseignement secondaire) mais un seul ministère pour les communautés flamande et germanophone. Par ailleurs, en Communauté flamande, les autorités responsables ont confié la gestion de la politique éducative à un organisme de droit public ${ }^{7}$ indépendant du ministère.

\section{Une organisation en réseau}

7 La notion de pouvoir organisateur (PO) est un élément fondamental dans l'organisation administrative du système éducatif belge. Le pouvoir organisateur représente toute personne physique ou morale assumant les responsabilités de création d'un établissement quel qu'il soit.

8 Trois réseaux d'enseignement ${ }^{8}$ existent à l'intérieur de chaque communauté, différenciés en fonction de leur pouvoir organisateur :

- un réseau organisé et financé sous l'autorité de la communauté fondatrice, c'est l'enseignement officiel de la communauté ;

- un réseau organisé par les provinces et les communes ${ }^{9}$ et subventionné par la communauté, c'est l'enseignement officiel subventionné ;

- un réseau d'enseignement libre, également subventionné par la Communauté mais organisé par des personnes privées ou des associations, confessionnelles ou non, c'est l'enseignement libre subventionné.

9 Ce dernier réseau est le plus fréquenté. Il est composé d'établissements catholiques à $95 \%{ }^{10}$ et représente presque la moitié des effectifs des élèves et des professeurs en 2000-2001 ${ }^{11}$. Les pouvoirs organisateurs se sont fédérés autour d'un organe de 
représentation, le secrétariat général de l'Enseignement catholique compétent pour toute négociation avec la Communauté française. dans les communautés et les régions. Pour ce qui concerne l'enseignement, il a été procédé à un partage au prorata du nombre d'élèves. C'est ainsi qu'a été déterminée pour la Communauté française une dotation ${ }^{13}$ figée qui n'a été modifiée qu'en 2001. A la différence de la Communauté flamande, qui dispose de sources de financement plus larges du fait de la fusion au niveau institutionnel des organes représentatifs de la communauté et de la région, la Communauté française n'a pas de moyens propres et dépend de la dotation de l'État fédéral. les établissements satisfont aux exigences des ministères de chaque communauté. Ces exigences concernent l'organisation des études et l'application des lois linguistiques. Les subventions publiques sont alors d'une triple nature: des subventions de traitement pour le personnel, des subventions de fonctionnement et des subventions pour la construction des locaux.

\section{L'organisation de la scolarité}

\section{Un cadre commun}

14 Bien que l'organisation des systèmes scolaires ait tendance à se différencier depuis la Communautarisation, la structure générale est identique dans chaque Communauté.

15 L'enseignement obligatoire dure douze ans, de six à dix-huit ans. Après l'enseignement fondamental, composé de l'enseignement maternel et primaire, l'enseignement secondaire se divise en trois niveaux de deux années chacun. Le premier niveau est un tronc commun à la fin duquel les élèves peuvent choisir entre quatre filières : générale, professionnelle, technique et artistique. Ceux qui ont achevé au moins les deux premières années de l'enseignement secondaire et qui ne désirent pas continuer à 
temps plein jusqu'à dix-huit ans peuvent alors suivre une formation obligatoire à temps partiel. Les élèves des trois communautés ayant réussi leur scolarité reçoivent un certificat de l'enseignement secondaire ${ }^{14}$. Toutes les certifications sont délivrées au niveau des établissements par les enseignants qui jugent leurs élèves sur la base de leur évaluation ${ }^{15}$.

En ce qui concerne la formation des enseignants, il n'existe pas de concours de recrutement des enseignants qui doivent chercher un poste dès l'obtention de leur diplôme à l'université. Les pouvoirs organisateurs disposent d'une grande liberté pour choisir leurs enseignants alors que le réseau officiel est soumis en matière de recrutement à plus de contraintes administratives ${ }^{16}$. Pour essayer d'enrayer la pénurie d'enseignants, les compétences acquises dans d'autres professions sont désormais prises en compte pour autoriser quelqu'un à enseigner.

\section{Diversité du fonctionnement}

17 En dépit de ce cadre commun aux trois communautés, c'est la diversité qui prime au sein des communautés en matière de fonctionnement des établissements. Les PO sont souverains et fixent par exemple le calendrier scolaire et la répartition de l'enseignement des disciplines. En Communauté francophone, le décret-mission du 24 juillet 1997 a fixé les missions prioritaires de l'enseignement fondamental et secondaire par le biais de l'élaboration d'un socle commun de règles identiques pour tous les établissements de chaque réseau. Il organise l'enseignement en cycles orientés par des compétences d'apprentissage. Ainsi, les six ans de l'école primaire sont divisés en trois cycles de deux années chacun pour lesquels ont été définis des socles de compétences à acquérir. Durant toute la durée du cycle, l'évaluation est continue et formative, un examen ayant lieu à la fin de chaque cycle pour vérifier que les compétences ont bien été assimilées. Des commissions de pilotage sont chargées de coordonner et de contrôler l'élaboration de ces socles, des programmes, tout en respectant les méthodes pédagogiques des $\mathrm{PO}$.

En Communauté flamande, la notion de compétence, Eindtermen, existe depuis 1996 sous une forme différente à chaque niveau d'enseignement. Cependant, plus qu'en Flandre peut-être, cette réforme a eu comme objectif premier de faire des économies budgétaires en diminuant les coûts et en réduisant le nombre d'échecs par la pratique du non redoublement.

\section{L'enseignement supérieur}

L'enseignement supérieur de type universitaire est dispensé dans seize établissements : six universités et dix établissements assimilés ${ }^{17}$. L'enseignement s'articule en trois cycles appelés respectivement la candidature, la licence et le doctorat. L'enseignement supérieur non universitaire, qui représente un taux très élevé de diplômés ${ }^{18}$, peut être court ou long en fonction des années d'étude et couvre huit filières (en Communauté française) et onze disciplines (en Communauté flamande). La Communauté germanophone ne dispose que d'un enseignement supérieur de type court. Les étudiants effectuent leurs études dans une des deux autres communautés de la Belgique ou en Allemagne. La majorité opte pour la Communauté française de Belgique. 
20 Le système d'accès à l'enseignement supérieur n'est pas limitatif, les élèves ayant achevé avec succès leurs études secondaires supérieures peuvent accéder à l'enseignement supérieur qu'il soit universitaire ou non. L'absence de numerus clausus, de tests d'entrée ou de systèmes d'orientation, permet à l'étudiant de choisir sa filière mais entraîne un taux d'échec très lourd à la fin de la première année.

\section{L'enseignement des langues}

21 L'enseignement des langues étrangères est très complexe en Belgique. Pour les trois communautés, la langue d'enseignement doit être celle de la région linguistique où se trouve l'école. Il est possible d'organiser dès l'enseignement primaire l'enseignement de la seconde langue qui est, en vertu de la loi linguistique du 30 juillet 1963 relative au régime linguistique de l'enseignement, le français en Communauté flamande et germanophone et le néerlandais en Communauté française. Dans la région de Bruxelles capitale, francophones et néerlandophones s'affrontent. En effet la ville de Bruxelles, officiellement bilingue, est majoritairement peuplée de francophones ${ }^{19}$ et enclavée dans le territoire flamand. D'après le « Pacte de courtoisie ", signé par les responsables politiques, il existe un enseignement réciproque des deux langues officielles dans les écoles, c'est-à-dire que les écoles flamandes enseignent le français en langue seconde et les écoles francophones enseignent le flamand comme langue seconde. Or les francophones de Bruxelles acceptent difficilement qu'on leur impose ce bilinguisme et remettent en cause l'enseignement du néerlandais arguant du manque de débouchés internationaux du néerlandais, contrairement au français. Les Flamands, déjà minoritaires, y voient une volonté de suprématie des francophones et une atteinte à leur identité.

\section{La liberté d'enseignement en Belgique : un principe fondateur}

En dépit d'une organisation différenciée en réseaux, l'enseignement en Belgique s'enracine dans un principe commun impliquant toute l'organisation générale du système éducatif belge. Évoquée pour la première fois dans la Constitution belge lorsque le pays accède à l'indépendance en 1830, la liberté de l'enseignement est, à l'époque, un compromis entre les partisans de la primauté de l'enseignement de l'État et ceux qui prônent sa non intervention ${ }^{20}$. Ce principe organise, de fait, la libre concurrence en matière d'enseignement. En 1959, la loi sur le Pacte scolaire apporte une nouvelle évolution à ce principe en consacrant le pluralisme des institutions. Ainsi tout pouvoir organisateur, qu'il fasse partie des pouvoirs publics ou non, est libre de créer son école moyennant le respect de certaines conditions. En outre, les parents ont le droit de choisir l'éducation qu'ils veulent pour leurs enfants, ce qui implique la possibilité de trouver, à une distance convenable, une école correspondant à leur choix, confessionnel ou non. D'un point de vue financier, tous les établissements d'enseignement reconnus viables obtiennent des subventions. Actuellement, en vertu du respect du libre choix des parents, chaque communauté a le droit de créer ou d'organiser des établissements, mais elle peut également en subventionner d'autres reconnus viables. En outre, elle est obligée d'organiser ou de subventionner un enseignement non confessionnel dans le cas où il n'en existerait pas à une distance 
convenable. Ainsi la liberté d'enseignement en Belgique implique la liberté de création des écoles, la liberté de choix des parents et la liberté pédagogique au sein des établissements, à condition de respecter le socle minimal prévu par la loi. Chaque pouvoir organisateur peut déterminer ses programmes, sous réserve de les soumettre à l'approbation ministérielle, évaluer et certifier ses élèves, définir un projet éducatif et pédagogique et recruter ses enseignants. Ce pacte consacre en outre le libre choix entre morale non confessionnelle et religion dans le système officiel ainsi que la gratuité de l'enseignement obligatoire pour tous les établissements.

\section{Évolution}

23 À l'origine, la liberté d'enseignement est d'ordre philosophique ${ }^{21}$ et vise à légitimer la présence de plusieurs communautés dans le domaine éducatif. Or ce principe évolue de plus en plus vers celui d'une liberté de marché avec un mécanisme de concurrence. Actuellement, les membres des communautés se positionnent en usagers de services évalués du point de vue de leurs qualités intrinsèques plutôt qu'en fonction de choix idéologiques. Dans ce contexte, les établissements évoluent dans un climat de concurrence accrue qui provoque de grandes disparités entre eux, non seulement au sein des communautés mais aussi dans les réseaux eux-mêmes ${ }^{22}$.

\section{Une plus grande rationalisation}

Les trois communautés ont vite été confrontées aux difficultés liées à cette autonomie des pouvoirs organisateurs sans pilotage de l'ensemble. La multiplication des établissements scolaires au sein des trois communautés et la dispersion des responsabilités entre les différents pouvoirs organisateurs ont rendu difficile un contrôle national global comme celui de l'obligation scolaire. Elle a entraîné également une situation coûteuse et difficilement maîtrisable concernant l'offre d'options par les différentes écoles ${ }^{23}$, chaque école faisant de la surenchère pour rallier un plus grand nombre d'élèves. Le manque de moyens en Communauté française ${ }^{24}$ empêche la mise en place de correctifs dans cet environnement concurrentiel et provoque cette importante différence de performances entre établissements soulignée par l'enquête PISA ${ }^{25}$.

\section{Un contrôle renforcé}

Devant ces difficultés, les pouvoirs publics ont soutenu une politique de rationalisation et une logique de concertation entre les différents acteurs éducatifs susceptibles d'atténuer la concurrence. Ainsi les trois communautés ont pris des mesures plus strictes $^{26}$. En 1993, des conseils de zone ont été créés. Ils sont chargés de vérifier l'offre d'options des établissements scolaires et peuvent refuser la création d'une école si ce qu'elle propose existe déjà. De même, un établissement peut être fermé s'il ne compte plus assez d'élèves: les normes de maintien et de création se renforcent au niveau des communautés. Quant au contrôle de l'obligation scolaire, il est lui, aussi, de mieux en mieux assuré. Dans la Communauté française, un système de carte d'inscription scolaire avec code barre a été instauré à la rentrée 1996. Ce système permet de comparer la population scolaire potentielle à la population scolaire effective et de retrouver les élèves considérés en décrochage scolaire. 


\section{Une meilleure concertation}

Depuis 1990, afin d'améliorer la concertation, tous les établissements sont tenus de mettre en place un conseil de participation qui réunit les pouvoirs organisateurs et les représentants des partenaires de l'école. Le conseil a pour rôle de se prononcer et d'évaluer le projet d'établissement et de remettre un avis sur le rapport d'activité annuel, élaboré par le pouvoir organisateur et remis à une commission de pilotage. De même à l'intérieur des établissements, les enseignants sont invités à travailler de manière plus collective et à développer un projet coordonné à l'intérieur des cycles de formation. Le décret de 1998 impose au personnel directeur et enseignant soixante heures de concertation annuelles. Parmi les mesures appliquées en 2003 qui s'inscrivent dans les mêmes perspectives, on peut signaler la création de la commission de pilotage du système éducatif en Communauté française.

\section{Orientations actuelles au niveau du secondaire et de l'enseignement supérieur}

Afin de favoriser l'hétérogénéité au sein des établissements scolaires, le ministre de l'Éducation fondamentale de la Belgique francophone propose un système de financement différenciée ${ }^{7}$ qui doit fonctionner depuis la rentrée 2004. Dans ce système, chaque élève issu d'un quartier défavorisé devrait permettre à l'établissement dans lequel il est inscrit de bénéficier d'une subvention. Ainsi, plus les établissements inscriront d'élèves issus de ces quartiers, plus les subventions seront élevées. Par ailleurs, à partir de la rentrée scolaire 2004, les écoles libres, autrefois en mesure de sélectionner leur public à l'inscription, sont désormais obligées d'accueillir tout élève qui le demande ${ }^{28}$. Le manque de place est désormais le seul motif légal pour refuser une demande d'inscription.

La mise en conformité de l'enseignement supérieur belge avec les exigences européennes fait partie des priorités du gouvernement. En 2004, l'agence pour l'évaluation de la qualité de l'enseignement supérieur a pris ses fonctions ${ }^{29}$. Cette agence, constituée de représentants d'université ou d'autres établissements d'enseignement supérieur, de personnel administratif, d'étudiants, de syndicats et des milieux économiques, doit fournir des observations concernant les pratiques pédagogiques, l'accueil et l'orientation des étudiants, la recherche et les partenariats.

\begin{tabular}{|l|l|l|}
\hline & Belgique & France \\
\hline Territoire & $32545 \mathrm{~km}^{2}$ & $547026 \mathrm{~km}^{2}$ \\
\hline Population & 10,3 Millions d'habitants & $59238000(2001)$ \\
\hline Densité & & $107,9 \mathrm{hab} / \mathrm{km}^{2}$ \\
\hline
\end{tabular}




\begin{tabular}{|l|l|l|}
\hline & $\begin{array}{l}58 \% \text { de néerlandophones } \\
32 \% \text { de francophones } \\
0,7 \% \text { de germanophones } \\
\begin{array}{l}9,3 \% \text { de bilingues dans l'agglomération } \\
\text { bruxelloise }\end{array}\end{array}$ & $\begin{array}{l}\text { Langue } \\
\text { française }\end{array}$ \\
\hline Nature de l'Etat & Monarchie constitutionnelle parlementaire & République \\
\hline PIB par habitant & 25000 (en 2000) & 22897 dollars \\
\hline $\begin{array}{l}\text { Dépense en éducation (en \% } \\
\text { PIB) }\end{array}$ & 5,9 & 5,8 \\
\hline
\end{tabular}

Le soutien de la cohésion nationale par la promotion de la mobilité étudiante intercommunautaire est l'objet d'un accord entre les trois ministres de l'Enseignement supérieur. Les ministres des trois communautés du pays ont donné le coup d'envoi d'un nouveau projet d'échange baptisé «Erasmus Belgique ${ }^{30}$. L'objectif est de donner aux étudiants la possibilité de suivre une partie de leur cursus au sein d'une institution reconnue d'une autre communauté tout en obtenant leur diplôme dans les délais prévus. L'organisation des premiers échanges est prévue en 2005 grâce à deux cent cinquante bourses.

Dans le même sens, le gouvernement a fait des propositions de création d'écoles bilingues franco-flamandes afin de développer de manière plus précoce pour les élèves une culture linguistique différente de celle de leur langue maternelle.

La situation socio-économique contraint la Belgique à une gestion plus rigoureuse du secteur éducatif. Or l'action des pouvoirs publics se heurte aux équilibres institutionnels délicats et aux arbitrages subtils entre les différents acteurs éducatifs. Ainsi, tout effort de rigueur doit tenir compte des marges d'autonomie auxquels sont attachés les pouvoirs organisateurs sous peine de devenir problématiques. La recherche de réponses adaptées aux aspirations légitimes mais parfois contradictoires de groupes et entités variés au sein d'un même territoire peut faire assimiler la Belgique à un laboratoire de l'Europe.

\section{BIBLIOGRAPHIE}

Michel et Raymonde Briquet, Le Système éducatif belge, CIEP, 1990.

Citron Pascaline, « En direct de Bruxelles », JDI, février 2000, nº.

Maroy Christian, Dupriez Vincent, «La régulation dans les systèmes scolaires », in Revue française de pédagogie, janvier-février-mars 2000, $\mathrm{n}^{\circ} 130$.

Élisabeth Thuriet, « L'école en Belgique », Les cahiers pédagogiques, n 425, juin 2004.

Francine Vaniscotte, Les écoles de l'Europe : systèmes éducatifs et dimension européenne, 1996, 350 p. 
«La Communauté française de Belgique », Confemen au quotidien, août-septembre 2002, nº 54 .

Reine-Marie Braken, « Le système éducatif en Communauté française », http://www.uvcw.be/ matieres/enseignement/index.cfm

\section{NOTES}

1. Eurydice, Belgique : la Communautarisation de l'enseignement, 1991.

2. Ministère de l'Education, Le système éducatif en Communauté française de Belgique, 1996, p. 11.

3. Les régions ont l'autorité sur l'économie, l'emploi et l'aménagement du territoire.

4. Michel Crahay, "Une école de qualité pour tous ", in http://www.wallonie-en-ligne.net/ wallonie-education/index.htm

5. idem, p. 13.

6. Les Flamands ont fondu les institutions de leur Région et de leur Communauté. L'ensemble comporte un Parlement et un gouvernement.

7. Le Conseil autonome de l'enseignement communautaire (ARGO) : il comprend des membres du gouvernement et d'autres membres élus.

8. «La Communauté française de Belgique ", in Confemen au quotidien, août-septembre 2002, $\mathrm{n}^{\circ}$ 54.

9. La Belgique est un État fédéral à trois échelons : région ou communauté, province, commune.

10. Il existe également des écoles protestantes, israélites et depuis 1989 une école islamique.

11. Élisabeth Thuriet, "L'école en Belgique » in Les cahiers pédagogiques, $n^{\circ} 425$, juin 2004, p. 49.

12. À titre de comparaison, en France, $21 \%$ de la population scolaire fréquente des établissements privés sous contrat, et seulement $1 \%$ en Finlande. Martine Vandermeulebroucke, «La liberté scolaire crée des inégalités », Le Soir, 21 juin 2003.

13. Élisabeth Thuriet, «L'école en Belgique » in Les cahiers pédagogiques, n 425, juin 2004, p. 52.

14. Francine Vaniscotte, Les écoles de l'Europe: systèmes éducatifs et dimension européenne, 1996, p. 149.

15. Élisabeth Thuriet, "L'école en Belgique », in Les cahiers pédagogiques, n 425, juin 2004, p. 49.

16. idem.

17. Francine Vaniscotte, p. 150

18. Élisabeth Thuriet, " L'école en Belgique » in Les cahiers pédagogiques, $n^{\circ} 425$, juin 2004, p. 53.

19. En 2000 , on comptait entre $65 \%$ et $70 \%$ de francophones et $10 \%$ de néerlandophones.

20. Reine-Marie Braken, " Le système éducatif en Communauté française ", in http:// www.uvcw.be/matieres/enseignement/index.cfm

21. Maroy Christian, Dupriez Vincent, "La régulation dans les systèmes scolaires ", in Revue française de pédagogie, janvier-février-mars 2000, n 130, p. 81.

22. Michel Crahay, http://www.wallonie-en-ligne.net/wallonie-education/index.htm.

23. « En 1992, il existait dans la Communauté française 6000 options comptant entre 1 et 5 élèves » En direct de Bruxelles, In JDI : Journal des Instituteurs p. 63.

24. Élisabeth Thuriet, «L'école en Belgique », in Les Cahiers pédagogiques, $\mathrm{n}^{\circ} 425$, juin 2004, p. 52.

25. PISA : Program for International Student Assessment.

26. Pascaline Citron, « En direct de Bruxelles », in JDI : Journal des Instituteurs, février 2000, $n^{\circ} 6$.

27. Pierre Bouillon, « Un enfant n'égalera plus un enfant ! », in Le Soir, 12 janvier 2004.

28. Pierre Bouillon, « Premier arrivé, premier inscrit?", in Le Soir, 5 février 2004.

29. «La Belgique se dote d'une évaluation externe », La lettre de l'Étudiant, 23 février 2004, nº 178.

30. Monique Baus, «Échanges d'étudiants entre communautés", in La libre Belgique, 4 février 2004. 
INDEX

Mots-clés : système éducatif

Index géographique : Belgique

\section{AUTEUR}

\section{CÉCILE DE BOUTTEMONT}

Documentaliste, centre de ressources documentaires du CIEP. 